\title{
LncRNA DLGAP1-AS2 Overexpression Associates with Gastric Tumorigenesis; A Promising Diagnostic and Therapeutic Target
}

\section{Rogayeh Soltani}

Higher Education Institute of Rab-Rashid

Mohammad Amini

Tabriz University of Medical Sciences

Marziyeh Mazaheri Moghaddam

Tabriz University of Medical Sciences

Asiyeh Jebelli

Higher Education Institute of Rab-Rashid

\section{Sahar Ahmadiyan}

Tabriz University of Medical Sciences

Negar Bidar

Tabriz University of Medical Sciences

\section{Behzad Baradaran}

Tabriz University of Medical Sciences

Habib MotieGhader

Islamic Azad University Tabriz Branch

Milad Asadi

Tabriz University of Medical Sciences

Ahad Mokhtarzadeh ( $\square$ ahad.mokhtarzadeh@gmail.com )

Tabriz University of Medical Sciences

\section{Research Article}

Keywords: Gastric cancer, LncRNA, DLGAP1-AS2, YAP1, Oncogene, TCGA

Posted Date: May 20th, 2021

DOI: https://doi.org/10.21203/rs.3.rs-529611/v1

License: (c) (1) This work is licensed under a Creative Commons Attribution 4.0 International License.

Read Full License 
Version of Record: A version of this preprint was published at Molecular Biology Reports on January 4th, 2022. See the published version at https://doi.org/10.1007/s11033-021-07038-w. 


\section{Abstract}

Background: Aberrant expression of long noncoding RNAs (IncRNAs) is associated with the progression of human cancers, including gastric cancer (GC). The function of IncRNA DLGAP1-AS2, as an oncogene, has been identified in glioma, hepatocellular carcinoma, and cholangiocarcinoma but not in other malignancies. Therefore, this study was aimed to explore the association of DLGAP1-AS2 with gastric tumorigenesis and beyond.

Methods and Results: The expression level of DLGAP1-AS2 was prevaluated in GC datasets from Gene Expression Omnibus (GEO). Moreover, qRT-PCR experiment was performed on 25 paired GC and adjacent normal tissue samples. The Cancer Genome Atlas (TCGA) data were also analyzed for further validations. Consistent with data obtained from GEO datasets, qRT-PCR results revealed that DLGAP1-AS2 was significantly $(p<0.0032)$ upregulated in $\mathrm{GC}$ specimens compared to normal samples, which was additionally confirmed using TCGA analysis $(\mathrm{p}<0.0001)$. DLGAP1-AS2 expression level was also correlated with age $(p=0.0008)$, lymphatic and vascular invasion $(p=0.0415)$ in internal samples. Also, a significant correlation was found between DLGAP1-AS2 and YAP1 expression, as its valid downstream target, in GC samples. Besides, analysis of other prevalent tumor entities using TCGA illustrated the significant overexpression of DLGAP1-AS2 in lung, colorectal, and prostate cancers, further indicating its promise as an oncogene. Moreover, ROC curve analysis showed the high accuracy of the DLGAP1-AS2 expression pattern as a diagnostic biomarker for gastric and colorectal cancers.

Conclusion: Our findings indicated that DLGAP1-AS2 might display oncogenic property in gastric tumorigenesis and be suggested as a therapeutic and diagnostic target.

\section{Introduction}

Gastric cancer (GC), also known as stomach cancer, is a major human health problem, with over one million new cases reported worldwide each year [1,2]. Although the GC incidence has been decreased recently, it remains the fifth most frequently diagnosed malignancy and accounts for the third cause of cancer-related death in the world [3, 4]. The GC cases are often diagnosed at an advanced stage, in which the surgery is not recommended, and the efficacy of treatment options, including chemotherapy and radiotherapy, are limited [5-7]. GC is a multifactorial disease in which genetic, epigenetic, and environmental factors have been identified to participate in GC development $[8,9]$.

Long non-coding RNAs (IncRNAs) are transcripts with lengths exceeding 200 nucleotides play a role in gene expression regulation [10]. A growing body of evidence has revealed that the aberrant expression of IncRNAs is involved in tumorigenesis, progression, and metastasis in numerous cancers, including GC [11-14]. Some IncRNAs such as PCAT18 and IINC01133 have low cont in GC patients; however, H19, CCAT1, and GHET1 are abundantly transcribed in GC samples [15-20]. Many studies have provided evidence indicating that several IncRNAs function as an oncogene or tumor-suppressor in GC $[21,15,19$, 22]. Besides, some IncRNAs have been revealed as potential biomarkers for GC diagnosis [23-25]. 
Further investigations of IncRNAs might help elucidate the molecular mechanisms involved in GC and provide clues that may help clinical application of IncRNAs in GC diagnosis [21].

Cytoplasmic IncRNA DLGAP1 antisense RNA 2 (DLGAP1-AS2), localized on human chromosome 18p11.31, is transcribed in the antisense of the DLGAP1 gene. The DLGAP1-AS2 function has been recently identified in glioma, hepatocellular carcinoma (HCC), and Cholangiocarcinoma (CCA) progression. However, little is known about tumorigenesis-related function of DLGAP1-AS2 [26-28]. To the best of our knowledge, the role of the DLGAP1-AS2 in GC progression has not been studied thus far. Therefore, we aimed to study the association of DLGAP1-AS2 with gastric tumorigenesis. In this regard, we initially analyzed microarray datasets from the Gene Expression Omnibus database (GEO) to evaluate the DLGAP1-AS2 expression level in GC samples. Subsequently, the outcomes were validated in GC and paired normal tissue samples and datasets from The Cancer Genome Atlas-Stomach Adenocarcinoma (TCGA-STAD) database. Also, the correlations between DLGAP1-AS2 expression level and clinicopathological features were analyzed.

\section{Material And Methods}

\subsection{In-silico investigation of DLGAP1-AS2 expression using the GEO database}

We first analyzed microarray expression datasets from the GEO (http://www.ncbi.nlm.nih.gov/geo/) database to identify the DLGAP1-AS2 expression level in GC compared to normal tissues. Three datasets were retrieved, including GSE79973 (10 GC and 10 paired normal tissues), GSE19826 (12 GC and its 12 adjacent noncancer tissues), and GSE54129 (111 GC tissues and 21 noncancerous gastric tissues). Differentially expressed genes between gastric tumors and noncancerous gastric samples were obtained using GEO2R available at http://www.ncbi.nlm.nih.gov/geo/geo2r/.

\subsection{Sample preparation}

After written informed consent, twenty-five GC samples and paired non-tumoral adjacent tissues were obtained from the Iranian tumor bank (Tehran, Iran). Tissue samples were stored in liquid nitrogen until RNA extraction. The enrolled subjects received no radiotherapy and chemotherapy before the surgery. Clinicopathological and epidemiological data assessed for this study include gender, age, tumor size, primary tumor site, lymphatic invasion, histological grade, perineural, serosal and vascular invasion, clinical-stage, smoking status, and GC family history.

\subsection{RNA extraction and CDNA synthesis}

Tissue specimens were ground in liquid nitrogen using a mortar and pestle, afterward transferred into the lysis buffer, and homogenized with a needle and syringe. The total RNA was isolated using the AllPrep DNA/RNA Kit (Germany, Qiagen) according to the manufacturer's protocol. The concentration and quality of the RNA samples were calculated by NanoDrop spectrophotometer (ThermoFisher Scientific Life 
Sciences, USA). The $1 \%$ agarose gel electrophoresis was carried out to assess the integrity of RNA. cDNA was synthesized from $1 \mu \mathrm{g}$ of total RNA using the PrimeScriptTM RT Reagent Kit (TaKaRa Bio, Japan) in a final volume of $20 \mathrm{ml}$ according to the manufacturer's instruction.

\subsection{Quantitative real-time PCR ( $\mathrm{qPCR}$ )}

qPCR was performed using the BioFACT ${ }^{\mathrm{TM}} 2 \mathrm{X}$ Real-Time PCR Master Mix (Korea) and gene-specific primer sets in a $10 \mathrm{ml}$ total reaction volume. qPCR was done by Step One Plus Real-Time PCR System (Applied Biosystems, USA) in three steps as follows: initial denaturing at $95^{\circ} \mathrm{C}$ for $15 \mathrm{~min}, 45$ cycles of denaturation at $95^{\circ} \mathrm{C}$ for $10 \mathrm{~s}$; primer annealing temperature at $60^{\circ} \mathrm{C}$ for $30 \mathrm{~s}$; and elongation at $72^{\circ} \mathrm{C}$ for $20 \mathrm{~s}$. Melting curves were obtained at the end of each run. GAPDH gene was used as a reference gene for data normalization. The $2^{-\triangle \triangle C t}$ method was used to calculate the relative expression level of DLGAP1-AS2 between groups. Table 1 summarizes primer sequences.

Table 1

Primer sequences used for qPCR.

\begin{tabular}{|lll|}
\hline Target & Forward (5' - 3' direction) & Reverse (5' $\mathbf{-} \mathbf{3}^{\prime}$ direction) \\
\hline DLGAP1-AS2 & CTAACTCCTGCCAACATCGT & CCTTTGTAAGAATCCACTTCAA \\
\hline GAPDH & AAGGTGAAGGTCGGAGTCAAC & GGGGTCATTGATGGCAACAA \\
\hline 2.5. Data Validation using TCGA datasets
\end{tabular}

In order to confirm the GEO datasets results and qPCR analysis in GC internal samples, The Cancer Genome Atlas- Stomach Adenocarcinoma (TCGA-STAD) database was analyzed. RNAseq gene expression data were retrieved from the UCSC Xena Functional Genomics Explorer [29] and analyzed. Furthermore, using TCGA-STAD dataset Kaplan-Meier survival analysis was performed to determine the relevance of DLGAP1-AS2 expression with the overall survival (OS) of GC patients. Also, the expression levels of DLGAP1-AS2 in other common cancers entities, including breast, lung, colorectal and prostate cancers, were evaluated. ROC analysis was performed to evaluate the potential of DLGAP1-AS2 expression as a diagnostic biomarker for distinguishing GC and normal cases.

\subsection{Statistical analysis}

GraphPad 6 Prism was applied to analyze the qPCR results and datasets and draw the graphs. Paired and unpaired t-test and Mann-Whitney test were performed to statistically analyze differences of DLGAP1-AS2 expression level between groups. All data are presented as mean \pm SEM (standard error of the mean). A value of $P<0.05$ was considered to be significant.

\section{Results}

\subsection{DLGAP1-AS2 high levels in GC samples retrieved from GEO}


We initially analyzed microarray datasets from the GEO to evaluate the DLGAP1-AS2 expression levels in GC samples compared to normal gastric tissues. As shown in Fig. 1, all the GSE79973, GSE19826, and GSE54129 datasets were in line with each other and revealed that DLGAP1-AS2 is significantly upregulated $(p<0.05, p<0.01$ and $p<0.0001$, respectively) in GC samples compared to normal ones; indicating its significance through gastric tumorigenesis.

\subsection{DLGAP1-AS2 upregulation in internal GC samples}

We performed a qPCR experiment using the $25 \mathrm{GC}$ tissue samples and 25 paired noncancerous gastric tissues. The qPCR analysis data revealed that DLGAP1-AS2 was significantly upregulated $(p<0.0032)$ in GC tissue specimens compared to paired normal samples (Fig. 2), which confirmed the results obtained from GEO datasets.

The correlation between the expression level of DLGAP1-AS2 and clinicopathological features in GC patients was analyzed. There was no correlation between DLGAP1-AS2 expression level and gender, tumor size, primary tumor site, histological grade, perineural and serosal invasion, clinical-stage, smoking status, and GC family history (Table 2). Interestingly, the relative expression level of DLGAP1-AS2 was significantly correlated with age $(p=0.0008)(\mathrm{Fig} .3 \mathrm{a})$, and lymphatic and vascular invasion $(p=0.0415)$ (Fig. 3b). 
Table 2

The correlations between DLGAP1-AS2 expression and clinicopathological features in GC patients.

\begin{tabular}{|c|c|c|}
\hline \multirow[t]{2}{*}{ Properties } & \multirow[t]{2}{*}{ No. of cases (\%) } & $p$-value \\
\hline & & DLGAP1-AS2 \\
\hline \multicolumn{3}{|l|}{ Age } \\
\hline$<60$ & $12(48 \%)$ & \multirow[t]{2}{*}{0.0008} \\
\hline$>=60$ & $13(52 \%)$ & \\
\hline \multicolumn{3}{|l|}{ Gender } \\
\hline Male & $21(84 \%)$ & \multirow[t]{2}{*}{0.3560} \\
\hline Female & $4(16 \%)$ & \\
\hline \multicolumn{3}{|c|}{ primary tumor site } \\
\hline Gastric Cardia & $4(16 \%)$ & \multirow[t]{3}{*}{0.3430} \\
\hline Antrum & $5(20 \%)$ & \\
\hline Stomach & $16(64 \%)$ & \\
\hline \multicolumn{3}{|c|}{ Tumor size (cm) } \\
\hline$<5$ & $11(44 \%)$ & \multirow[t]{3}{*}{0.8700} \\
\hline$\geq 5$ & $12(48 \%)$ & \\
\hline Unknown & $2(8 \%)$ & \\
\hline \multicolumn{3}{|l|}{ Histology grade } \\
\hline I, II & $17(68 \%)$ & \multirow[t]{2}{*}{0.2303} \\
\hline III, IV & $8(32 \%)$ & \\
\hline \multicolumn{3}{|c|}{ Lymphatic invasion } \\
\hline Yes & $19(76 \%)$ & \multirow[t]{2}{*}{0.0415} \\
\hline No & $6(24 \%)$ & \\
\hline \multicolumn{3}{|c|}{ Vascular invasion } \\
\hline Yes & $19(76 \%)$ & \multirow[t]{2}{*}{0.0415} \\
\hline No & $6(24 \%)$ & \\
\hline \multicolumn{3}{|c|}{ Serosal invasion } \\
\hline Yes & $14(56 \%)$ & 0.1270 \\
\hline
\end{tabular}




\begin{tabular}{|c|c|c|}
\hline \multirow[t]{2}{*}{ Properties } & \multirow[t]{2}{*}{ No. of cases (\%) } & p-value \\
\hline & & DLGAP1-AS2 \\
\hline No & $11(44 \%)$ & \\
\hline \multicolumn{3}{|c|}{ Perineural invasion } \\
\hline Yes & 16 (64\%) & \multirow[t]{2}{*}{0.1928} \\
\hline No & $9(36 \%)$ & \\
\hline \multicolumn{3}{|c|}{ Clinical Stage } \\
\hline I, II & $9(36 \%)$ & \multirow[t]{2}{*}{0.0640} \\
\hline III, IV & 16 (64\%) & \\
\hline \multicolumn{3}{|c|}{ Family history } \\
\hline Yes & $9(36 \%)$ & \multirow[t]{2}{*}{0.7671} \\
\hline No & $16(64 \%)$ & \\
\hline \multicolumn{3}{|l|}{ Smoking } \\
\hline Yes & $14(56 \%)$ & \multirow[t]{2}{*}{0.5899} \\
\hline No & $11(44 \%)$ & \\
\hline
\end{tabular}

\subsection{Validation of DLGAP1-AS2 overexpression in TCGA- STAD dataset}

The TCGA-STAD database was also analyzed to confirm the results obtained from the GEO datasets and qPCR experiment. Overall, DLGAP1-AS2 expression was analyzed in $375 \mathrm{GC}$ and 32 normal samples. The data from TCGA-STAD analysis demonstrated that DLGAP1-AS2 is significantly overexpressed $(p<$ 0.0001) in GC samples compared to normal specimens (Fig. 4a). Kaplan-Meier survival analysis of GC patients further revealed no association between survival with the DLGAP1-AS2 expression level ( $p=$ 0.8172) (Fig. 4b). Moreover, as illustrated in Fig. 4c, DLGAP1-AS2 expression possesses the potential as a diagnostic target for distinguishing GC and normal samples with the AUC up to $0.89(p<0.0001)$.

\subsection{DLGAP1-AS2 expression was correlated with YAP1 levels in GC samples}

We further analyzed the association of DLGAP1-AS2 expression with YAP1 (Yes1 Associated Transcriptional Regulator) levels, as its valid downstream mediator, in GC patient's cohort from TCGA dataset. As shown in Fig. 5, Pearson's correlation analysis revealed a significant $(p<0.0001)$ positive 
correlation between DLGAP1-AS2 and YAP1 expression in GC samples $(\mathrm{r}=0.2623)$, which suggested that $D L G A P 1-A S 2$ may be involved in gastric tumorigenesis through modulating YAP1 expression.

\subsection{DLGAP1-AS2 expression levels in other prevalent cancers}

Considering that the involvement in DLGAP1-AS2 dysregulation hasn't been investigated in a wide array of human malignancies, its expression levels were evaluated in other common cancer entities using TCGA. The obtained results (Fig. 6a) illustrated that despite no significant difference in breast cancer samples, DLGAP1-AS2 exhibits high levels of expression in lung adenocarcinoma $(p<0.0001)$, colorectal $(p<0.0001)$, and prostate $(p<0.05)$ cancers compared to normal samples. Besides, as illustrated in Fig. $6 \mathrm{~b}, \mathrm{ROC}$ curve analysis showed a high diagnostic value of DLGAP1-AS2 colorectal cancer as well ( $p<$ 0.0001 , and $A U C=0.95$ ); suggesting its great promise as a diagnostic marker for gastrointestinal malignancies.

\section{Discussion}

Despite the decreased incidence of GC, it is still a major human health concern. Exploration of pathogenic molecular mechanisms underlying GC is still needed for early detection of GC and decline its mortality rates [30]. Since IncRNAs are involved in gene regulation, they have received increasing attention recently [21]. Additionally, aberrant expression of IncRNAs is associated with the development and progression of numerous cancers, including GC $[21,24]$.

Accordingly, to study the association of DLGAP1-AS2 with gastric tumorigenesis, we first analyzed microarray expression datasets from the GEO database to evaluate the DLGAP1-AS2 expression level in GC and normal tissues. DLGAP1-AS2 expression was found to be elevated in GC cases compared to normal samples. Also, the qPCR analysis data revealed that DLGAP1-AS2 was significantly upregulated in GC tissue specimens compared to paired normal samples. Another layer of confirmation came from analyzing the TCGA-STAD dataset. An interesting and unexpected finding was the negative correlation between DLGAP1-AS2 expression level and the patients' age, which is controversial and needs further investigation. Also, the relative expression level of DLGAP1-AS2 in GC tissue samples was significantly correlated with lymphatic and vascular invasion. Therefore, our findings indicate that the DLGAP1-AS2 overexpression might be associated with GC progression and metastasis and suggest the possible oncogenic role of DLGAP1-AS2 in GC as well.

Additionally, further analysis DLGAP1-AS2 expression in other prevalent malignancies using TCGA, illustrated this IncRNA is also upregulated in breast, colorectal, and prostate cancer specimens compared to normal samples, indicating a promising role of DLGAP1-AS2 as an oncogene through tumorigenesis of human cancers. In line with our findings, Liu et al. observed the up-regulation of DLGAP1-AS2 in CCA cell lines. Additionally, they found that DLGAP1-AS2 contributes to CCA progression by modulating the miR505/GALNT10 cascade [28]. Recently the overexpression of DLGAP1-AS2 and down-regulation of miR- 
154-5P was observed in patients with HCC. Further investigations identified that DLGAP1-AS2 knockdown in HCC cells resulted in decreased methylation and overexpression of miR-154-5p, consequently inhibition of HCC cell invasion and migration. Therefore, these findings provided clues that DLGAP1-AS2 might play a role as an oncogene in HCC [27]. Miao et al. found that DLGAP1-AS2 was overexpressed in glioma patients. They revealed that DLGAP1-AS2 depletion in glioma cells promoted cell apoptosis and inhibited cell proliferation as well as cell migration, which consequently attenuated the progression of glioma. Also, YAP1 expression was downregulated in these cells. Thereby, YAP1 was considered a downstream target of DLGAP1-AS2 and appears to play a biological role in glioma progression. These findings thus support the view that DLGAP1-AS2 might function as an oncogene in glioma progression [26]. Several lines of evidence from different studies have identified $Y A P 1$ as an oncogene in numerous cancers. Besides, overexpression of YAP1 has been identified in GC. Further investigations have revealed that up-regulation of YAP1 could promote cell proliferation, growth, and migration in GC and associates with the progression and lymph node metastasis of $\mathrm{GC}$ as well. Consequently, these findings demonstrate that YAP1 functions as an oncogene in GC [31-35].

It is noteworthy that we found a significant correlation between DLGAP1-AS2 and YAP1 expression in TCGA dataset by Pearson's correlation analysis. According to the previous studies and the correlation between DLGAP1-AS2 and YAP1 expression, YAP1 might be the downstream target of DLGAP1-AS2 in GC progression and invasion. Further investigations are required to identify the molecular mechanisms by which DLGAP1-AS2 contributes to the GC progression. According to the ROC curve analysis, DLGAP1-AS2 overexpression may be suggested as a potential diagnostic target for discriminating GC and normal cases $(p<0.0001, A U C=0.8920)$.

\section{Conclusion}

In conclusion, our results revealed that the aberrant overexpression of DLGAP1-AS2 might be associated with GC progression and metastasis and suggest the possible oncogenic role of DLGAP1-AS2 in GC as well. Also, YAP1 might be the downstream target of DLGAP1-AS2 in GC progression and lymphatic and vascular invasion. According to the ROC curve analysis, DLGAP1-AS2 may be suggested as a potential diagnostic target for GC. Also, our results illustrated overexpression of DLGAP1-AS2 in other prevalent malignancies, including lung, colorectal, and prostate cancers. However, further investigations could elucidate the molecular mechanisms by which DLGAP1-AS2 participates in tumorigenesis and provide clues that may help its clinical application.

\section{Declarations}

\section{Funding}

The authors are grateful for supports from the Immunology Research Center, Tabriz University of Medical Sciences, Tabriz, Iran. 


\section{Conflicts of interests}

The authors declare no conflict of interest.

\section{Availability of data and material}

All data generated in this study are available in the manuscript.

\section{Code availability}

Not applicable

\section{Authors' contributions}

R.S. and M.AM. performed the majority of experiments and data analysis. S.A. contributed to carry out the experiments and interpreted the results; M.AM. and H.B. helped with project design; M.M.M and N.B wrote the manuscript. B.B. and A.J. contributed to design the project; M. AS. helped with sample preparation. A.M. revised the manuscript, designed and conducted the project.

\section{Ethical approval}

The study was approved by the ethical committee of Tabriz University of Medical Sciences, Tabriz, Iran.

\section{Consent to participate}

All of the patients participated in the study had given written informed consent.

\section{Consent for publication}

Patients signed informed consent regarding publishing their data.

\section{References}

1. Thrift AP, El-Serag HB (2020) Burden of Gastric Cancer. Clin Gastroenterol Hepatol 18(3):534-542. doi:https://doi.org/10.1016/j.cgh.2019.07.045

2. Necula L, Matei L, Dragu D, Neagu Al, Mambet C, Nedeianu S et al (2019) Recent advances in gastric cancer early diagnosis. World journal of gastroenterology 25(17):2029-2044. doi:10.3748/wjg.v25.i17.2029

3. Bray F, Ferlay J, Soerjomataram I, Siegel RL, Torre LA, Jemal A (2018) Global cancer statistics 2018: GLOBOCAN estimates of incidence and mortality worldwide for 36 cancers in 185 countries. Cancer J Clin 68(6):394-424. doi:10.3322/caac.21492

4. Sitarz R, Skierucha M, Mielko J, Offerhaus GJA, Maciejewski R, Polkowski WP (2018) Gastric cancer: epidemiology, prevention, classification, and treatment. Cancer management research 10:239-248. doi:10.2147/cmar.s149619 
5. Marin J, Al-Abdulla R, Lozano E, Briz O, Bujanda L, Banales M J et al. Mechanisms of resistance to chemotherapy in gastric cancer. Anti-Cancer Agents in Medicinal Chemistry (Formerly Current Medicinal Chemistry-Anti-Cancer Agents). 2016;16(3):318-34

6. Cuzzuol BR, Vieira ES, Araújo GRL, Apolonio JS, de Carvalho LS, da Silva Junior RT et al. Gastric Cancer: A Brief Review, from Risk Factors to Treatment. Archives of Gastroenterology Research. 2020;1(2)

7. Shi WJ, Gao JB (2016) Molecular mechanisms of chemoresistance in gastric cancer. World J Gastrointest Oncol 8(9):673-681. doi:10.4251/wjgo.v8.i9.673

8. Amini M, Ghorban K, Mokhtarzadeh A, Dadmanesh M, Baradaran B (2020) CD40 DNA hypermethylation in primary gastric tumors; as a novel diagnostic biomarker. Life Sci 254:117774. doi:https://doi.org/10.1016/j.Ifs.2020.117774

9. Padmanabhan N, Ushijima T, Tan P (2017) How to stomach an epigenetic insult: the gastric cancer epigenome. Nature reviews Gastroenterology hepatology 14(8):467

10. Nasrollahzadeh-Khakiani M, Emadi-Baygi M, Schulz WA, Nikpour P (2016) Long noncoding RNAs in gastric cancer carcinogenesis and metastasis. Briefings in Functional Genomics 16(3):129-145. doi:10.1093/bfgp/elw011

11. Xiao N, Hu Y, Juan L (2020) Comprehensive analysis of differentially expressed IncRNAs in gastric cancer. Frontiers in Cell Developmental Biology 8:557

12. Tan $\mathrm{H}$, Zhang $\mathrm{S}$, Zhang J, Zhu L, Chen $\mathrm{Y}$, Yang $\mathrm{H}$ et al (2020) Long non-coding RNAs in gastric cancer: New emerging biological functions and therapeutic implications. Theranostics 10(19):8880

13. Li H, Yu B, Li J, Su L, Yan M, Zhu Z et al (2014) Overexpression of IncRNA H19 enhances carcinogenesis and metastasis of gastric cancer. Oncotarget 5(8):2318

14. Li T, Mo X, Fu L, Xiao B, Guo J (2016) Molecular mechanisms of long noncoding RNAs on gastric cancer. Oncotarget 7(8):8601-8612. doi:10.18632/oncotarget.6926

15. Yang F, Bi J, Xue X, Zheng L, Zhi K, Hua J et al (2012) Up-regulated long non-coding RNA H19 contributes to proliferation of gastric cancer cells. FEBS J 279(17):3159-3165. doi:10.1111/j.17424658.2012.08694.x

16. Zhang EB, Han L, Yin DD, Kong R, De W, Chen J (2014) c-Myc-induced, long, noncoding H19 affects cell proliferation and predicts a poor prognosis in patients with gastric cancer. Med Oncol (Northwood Lond Engl) 31(5):914. doi:10.1007/s12032-014-0914-7

17. Foroughi K, Amini M, Atashi A, Mahmoodzadeh H, Hamann U, Manoochehri M. Tissue-Specific Down-Regulation of the Long Non-Coding RNAs PCAT18 and LINC01133 in Gastric Cancer Development. Int J Mol Sci. 2018;19(12). doi:10.3390/ijms19123881

18. Zhuang M, Gao W, Xu J, Wang P, Shu Y. The long non-coding RNA H19-derived miR-675 modulates human gastric cancer cell proliferation by targeting tumor suppressor RUNX1. Biochemical and biophysical research communications. 2014;448(3):315-22. doi:10.1016/j.bbrc.2013.12.126

19. Yang F, Xue X, Bi J, Zheng L, Zhi K, Gu Y et al (2013) Long noncoding RNA CCAT1, which could be activated by c-Myc, promotes the progression of gastric carcinoma. J Cancer Res Clin Oncol 
139(3):437-445. doi:10.1007/s00432-012-1324-x

20. Yang F, Xue X, Zheng L, Bi J, Zhou Y, Zhi K et al (2014) Long non-coding RNA GHET1 promotes gastric carcinoma cell proliferation by increasing c-Myc mRNA stability. FEBS J 281(3):802-813. doi:10.1111/febs. 12625

21. Fang $X Y$, Pan HF, Leng RX, Ye DQ. Long noncoding RNAs: novel insights into gastric cancer. Cancer letters. 2015;356(2 Pt B):357 - 66. doi:10.1016/j.canlet.2014.11.005

22. Park SM, Park SJ, Kim HJ, Kwon OH, Kang TW, Sohn HA et al (2013) A known expressed sequence tag, BM742401, is a potent lincRNA inhibiting cancer metastasis. Exp Mol Med 45(7):e31. doi:10.1038/emm.2013.59

23. Liu H, Wu N, Zhang Z, Zhong X, Zhang H, Guo H et al (2019) Long non-coding RNA LINC00941 as a potential biomarker promotes the proliferation and metastasis of gastric cancer. Frontiers in genetics 10:5

24. Yang Z, Guo X, Li G, Shi Y, Li L (2016) Long noncoding RNAs as potential biomarkers in gastric cancer: Opportunities and challenges. Cancer letters 371(1):62-70. doi:https://doi.org/10.1016/j.canlet.2015.11.011

25. Li J, Xu Q, Wang W, Sun S. MIR100HG: a credible prognostic biomarker and an oncogenic IncRNA in gastric cancer. Bioscience reports. 2019;39(4)

26. Miao W, Li N, Gu B, Yi G, Su Z, Cheng H (2020) LncRNA DLGAP1-AS2 modulates glioma development by up-regulating YAP1 expression. The Journal of Biochemistry 167(4):411-418

27. Chen K, Zhang Z, Yu A, Li J, Liu J, Zhang X. IncRNA DLGAP1-AS2 Knockdown Inhibits Hepatocellular Carcinoma Cell Migration and Invasion by Regulating miR-154-5p Methylation. BioMed Research International. 2020;2020

28. Liu Z, Pan L, Yan X, Duan X (2020) The long noncoding RNA DLGAP1-AS2 facilitates cholangiocarcinoma progression via miR-505 and GALNT10. FEBS open bio. doi:10.1002/22115463.13061

29. Goldman M, Craft B, Swatloski T, Cline M, Morozova O, Diekhans M et al (2014) The UCSC Cancer Genomics Browser: update 2015. Nucleic Acids Res 43(D1):D812-D817. doi:10.1093/nar/gku1073

30. Matsuoka T, Yashiro M (2018) Biomarkers of gastric cancer: Current topics and future perspective. World journal of gastroenterology 24(26):2818-2832. doi:10.3748/wjg.v24.i26.2818

31. Yu L, Gao C, Feng B, Wang L, Tian X, Wang H et al (2017) Distinct prognostic values of YAP1 in gastric cancer. Tumour biology: the journal of the International Society for Oncodevelopmental Biology Medicine 39(4):1010428317695926. doi:10.1177/1010428317695926

32. Sun D, Li X, He Y, Li W, Wang Y, Wang H et al (2016) YAP1 enhances cell proliferation, migration, and invasion of gastric cancer in vitro and in vivo. Oncotarget 7(49):81062-81076. doi:10.18632/oncotarget.13188

33. Kang W, Tong JH, Chan AW, Lee TL, Lung RW, Leung PP et al (2011) Yes-associated protein 1 exhibits oncogenic property in gastric cancer and its nuclear accumulation associates with poor 
prognosis. Clinical cancer research: an official journal of the American Association for Cancer Research 17(8):2130-2139. doi:10.1158/1078-0432.ccr-10-2467

34. Liu J, Zhao X, Wang K, Zhang X, Yu Y, Lv Y et al (2019) A novel YAP1/SLC35B4 regulatory axis contributes to proliferation and progression of gastric carcinoma. Cell Death Dis 10(6):452. doi:10.1038/s41419-019-1674-2

35. Hu X, Xin Y, Xiao Y, Zhao J (2014) Overexpression of YAP1 is Correlated with Progression, Metastasis and Poor Prognosis in Patients with Gastric Carcinoma. Pathology Oncology Research 20(4):805811. doi:10.1007/s12253-014-9757-y

\section{Figures}

a

GSE79973

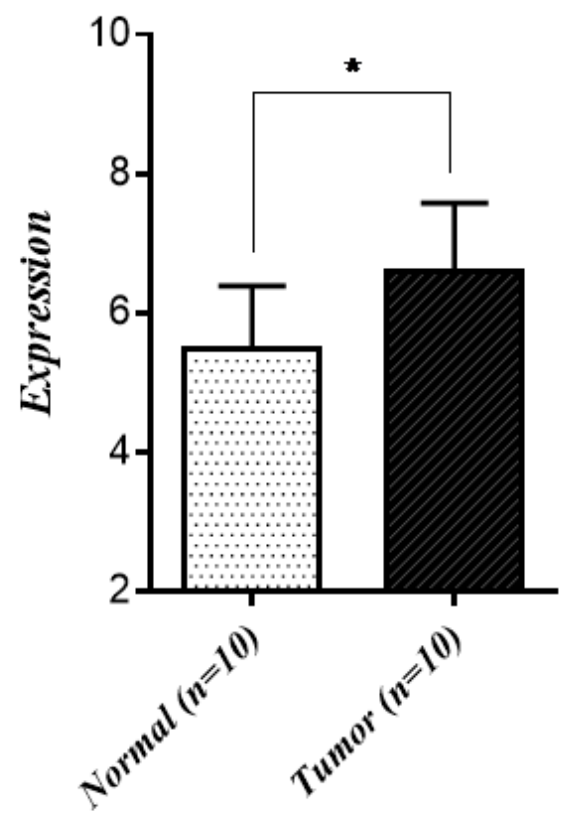

b

GSE19826

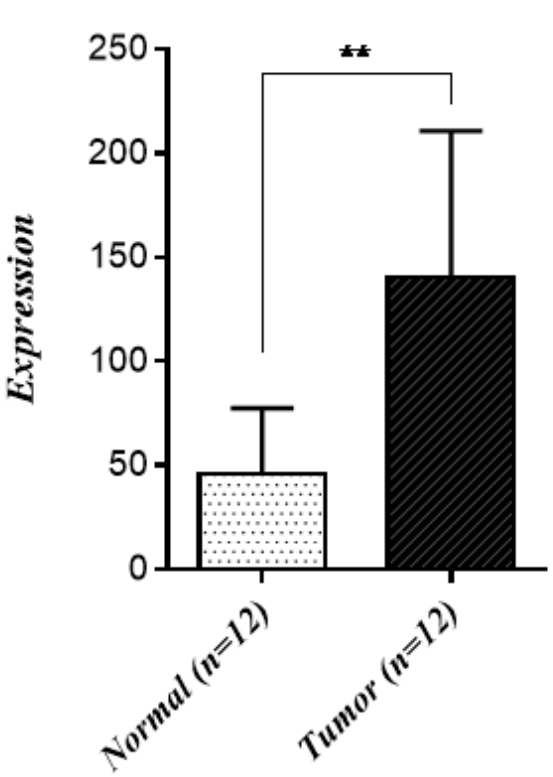

C

GSE54129

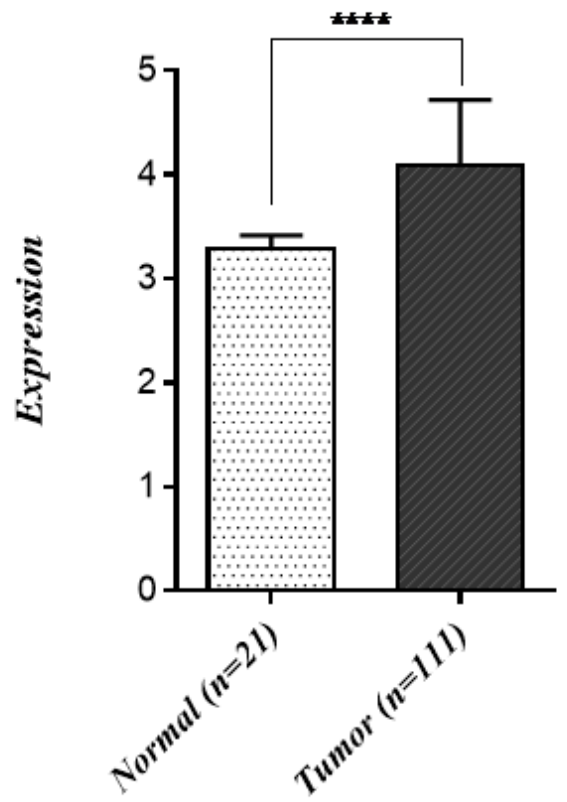

Figure 1

Investigation of DLGAP1-AS2 expression applying three GEO datasets in GC and normal cases (a) GSE79973, (b) GSE19826, and (c) GSE54129. * $p<0.05$, ** $p<0.01$, and *** $p<0.0001$. 


\section{DLGAP1-AS2}

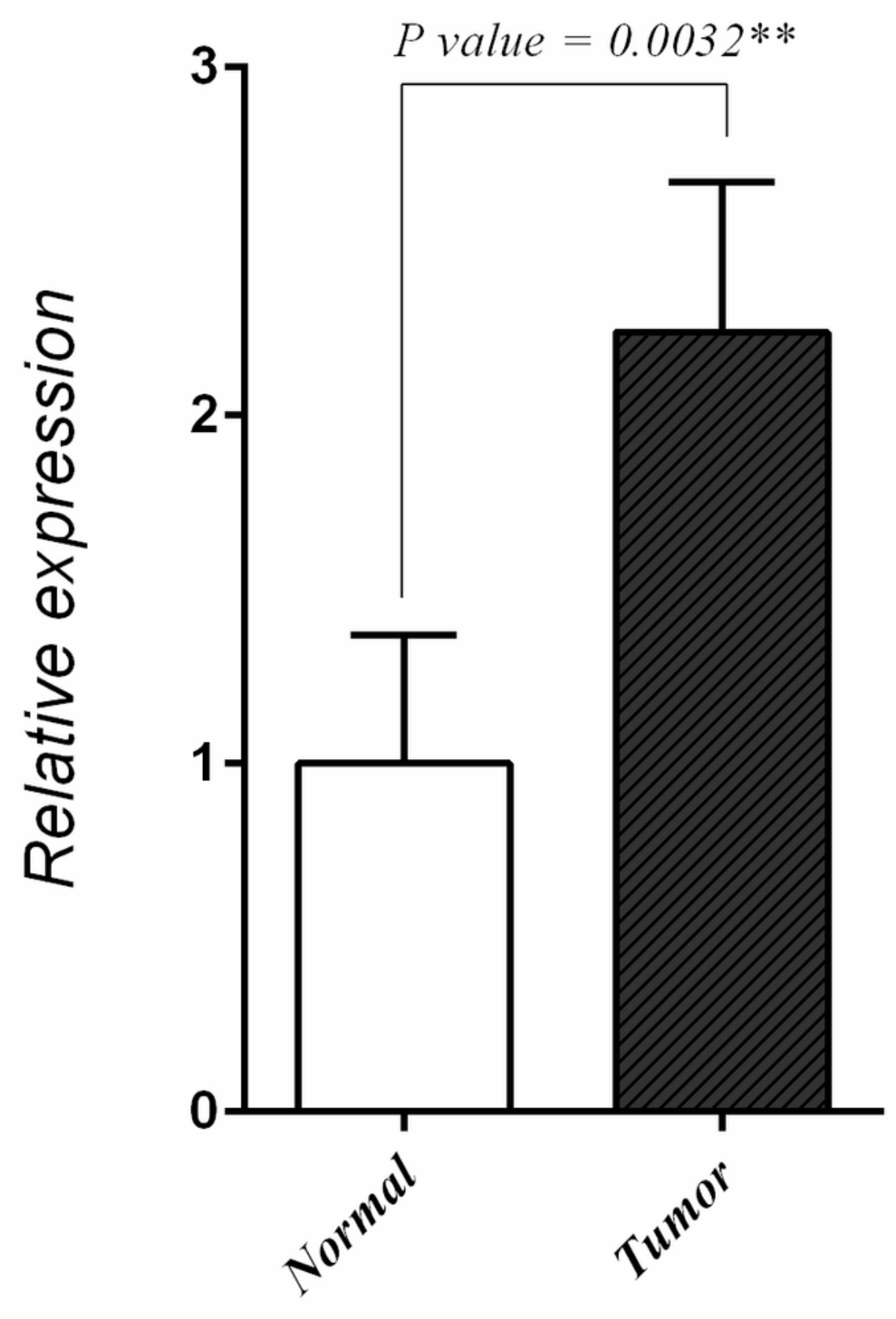

Figure 2

q-PCR analysis of DLGAP1-AS2 expression applying control tissue specimens $(\mathrm{N}=25)$ and gastric carcinoma cases $(\mathrm{N}=25) ; \mathrm{p}=0.0032$. 
a

Age

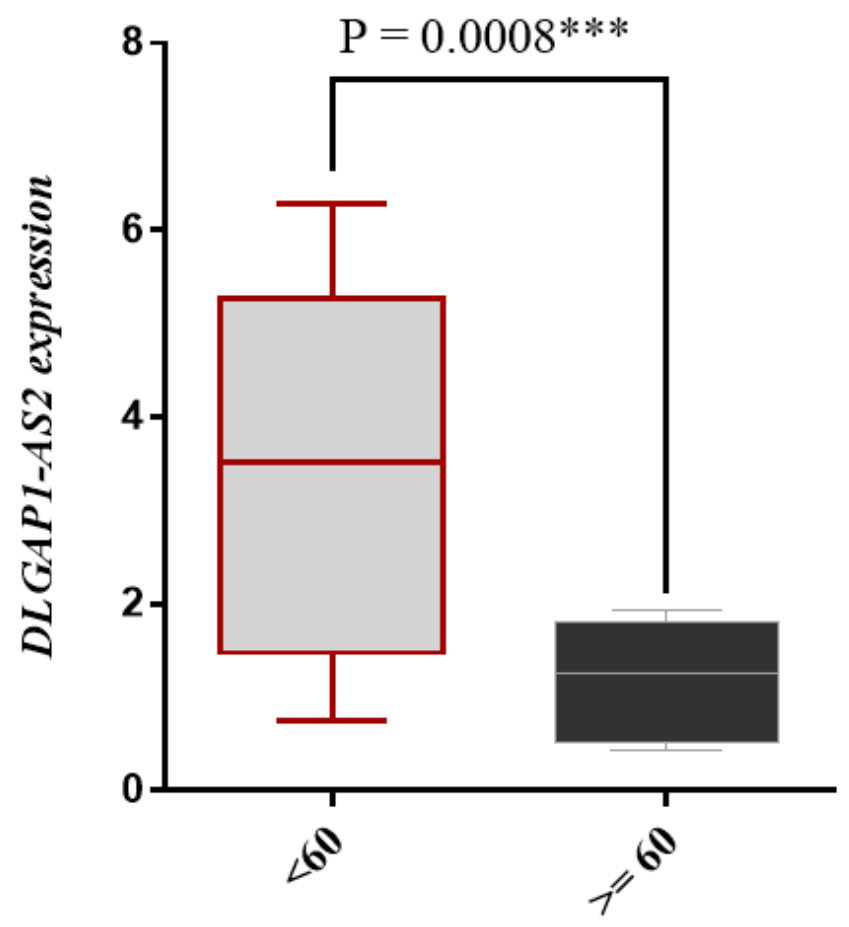

Groups b Lymphatic and vascular invasion

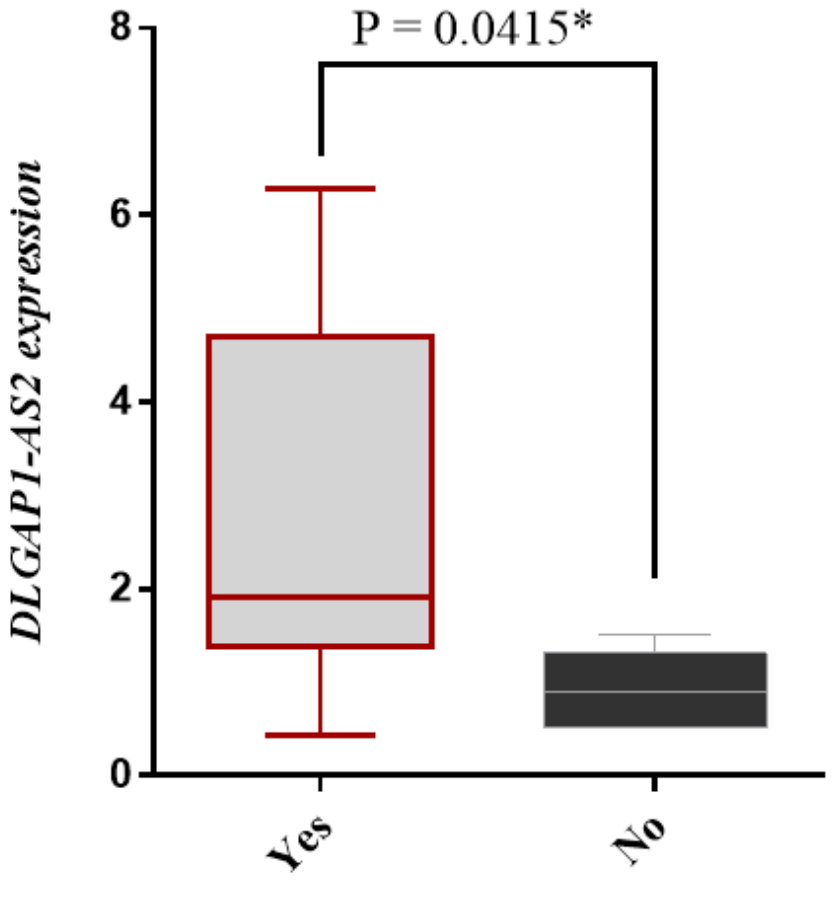

Groups

Figure 3

a) The correlations between DLGAP1-AS2 expression and GC patients age ( $* \star * p=0.0008)$. b) Lymph node status in GC tissue specimens ( $\left.{ }^{\star} p=0.0415\right)$. 

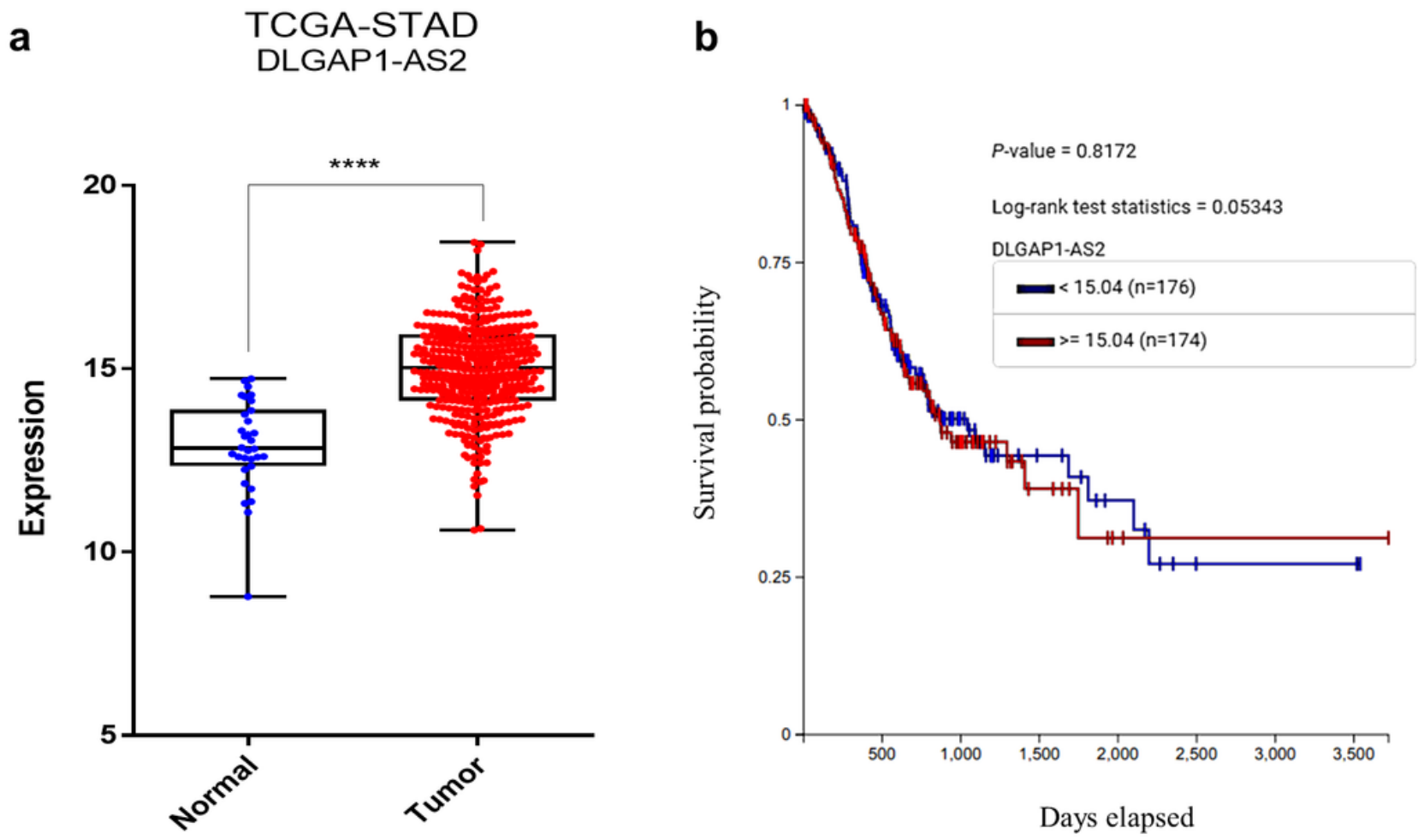

C ROC curve of DLGAP1-AS2

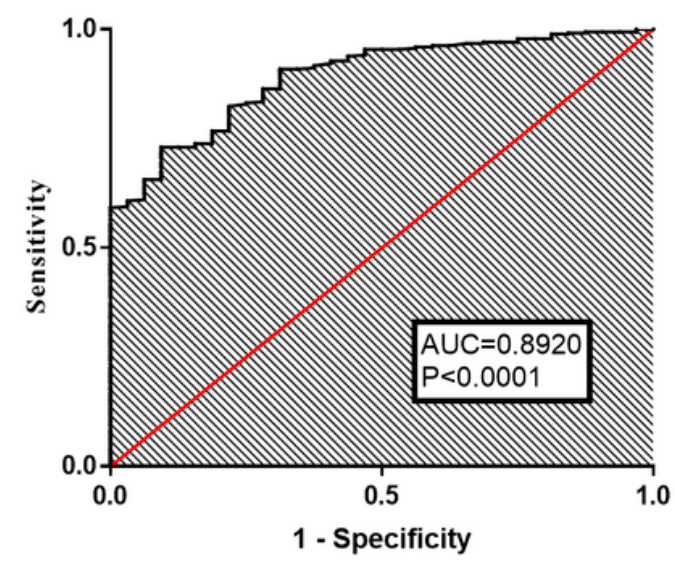

Figure 4

a) The validation of DLGAP1-AS2 overexpression in TCGA-STAD dataset; $* \star \star \star p<0.0001$. b) Comparison of the DLGAP1-AS2 overexpression and survival distribution $(p=0.8172)$. $c)$ ROC curve analysis for DLGAP1-AS2 overexpression was used to differentiate between GC and normal cases $(p<0.0001$, and $A U C=0.8920)$. 


\section{Pearson correlation}

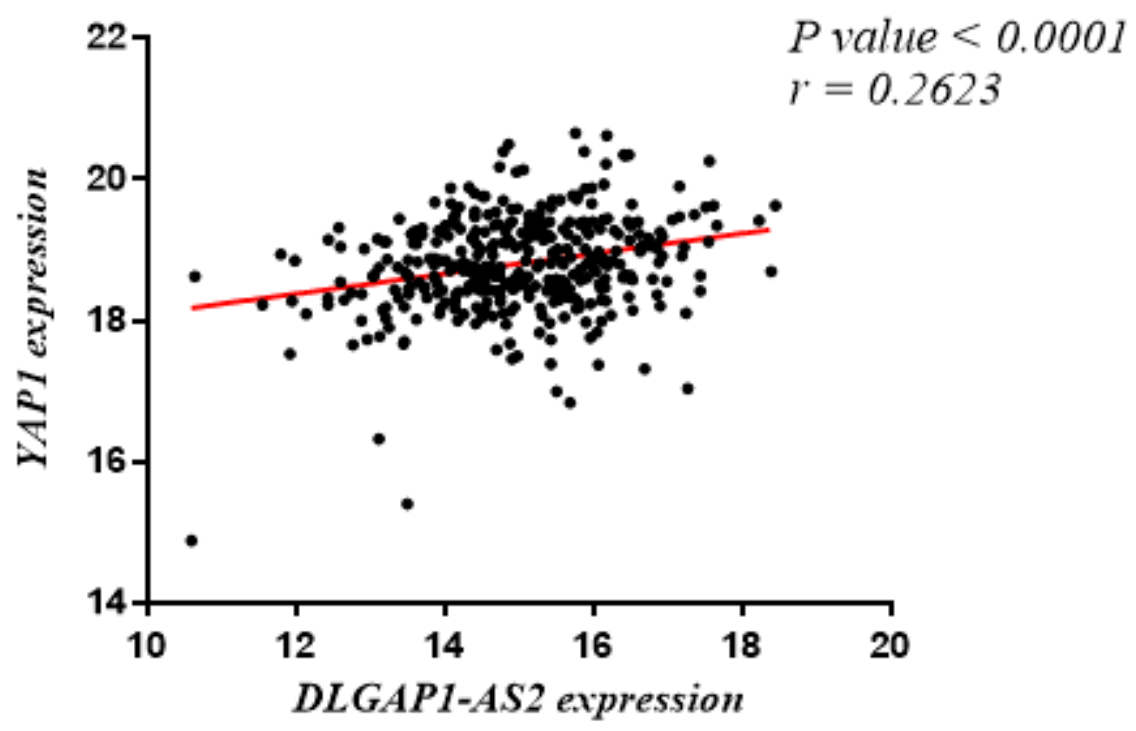

Figure 5

The correlation between DLGAP1-AS2 and YAP1 expression levels was analyzed using TCGA-STAD patient cohort. 
a
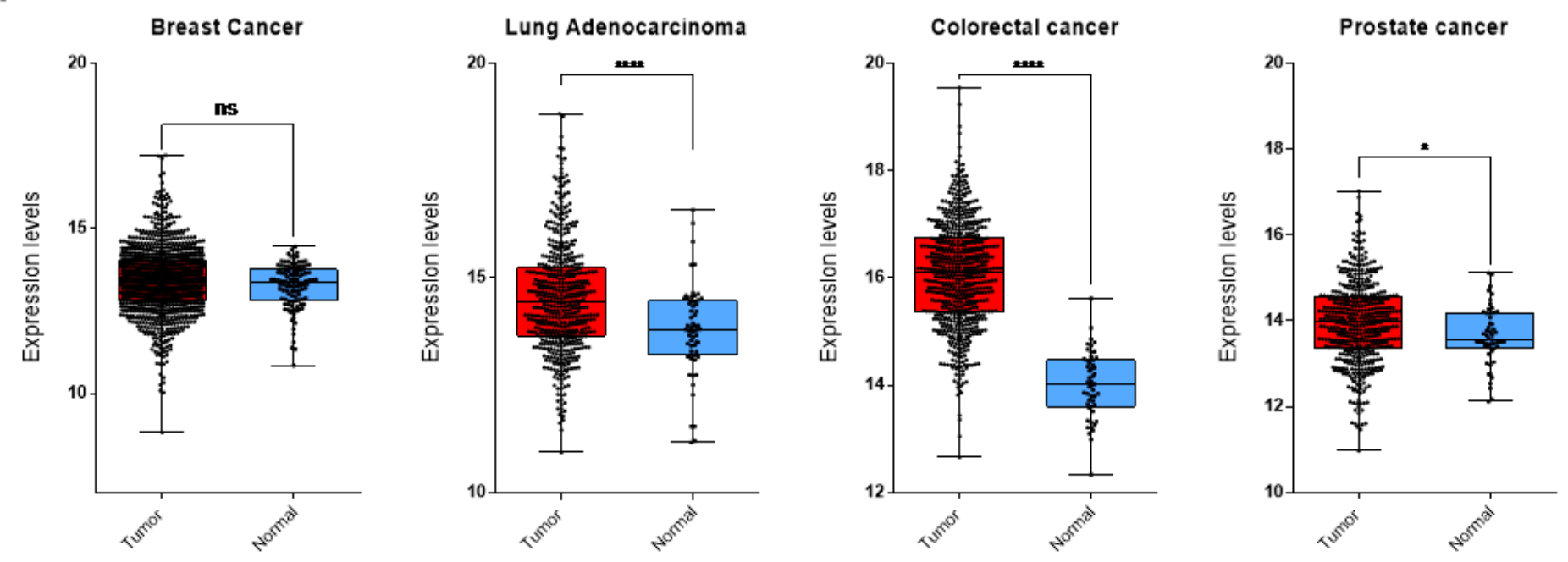

b
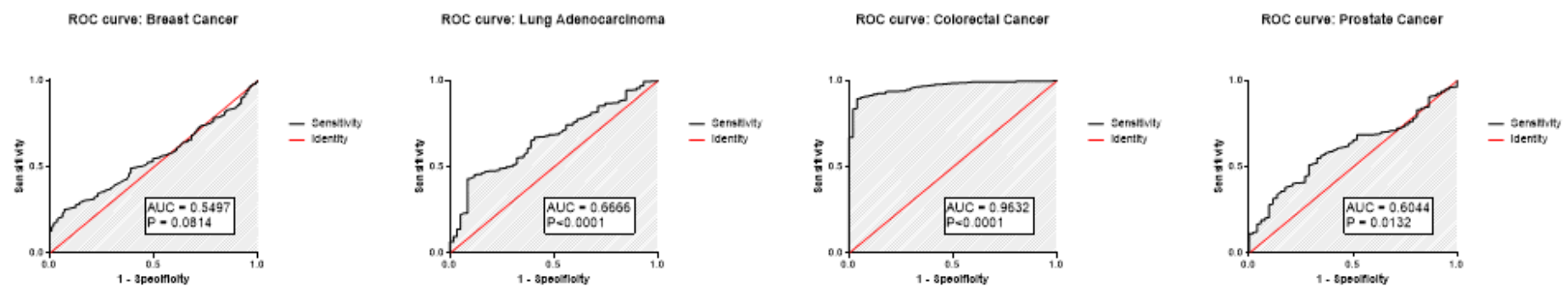

\section{Figure 6}

DLGAP1-AS2 status in other prevalent cancer entities using TCGA. a) DLGAP1-AS2 expression levels were analyzed in breast, lung, colorectal and prostate cancers. b) ROC curve analysis was performed. $\star \star \star \star p<0.0001,{ }^{*} p<0.01, \mathrm{~ns}=$ non-significant. 